\title{
An Innovative Approach to a Hybrid Quadrotor Design
}

\author{
Leroy Salvius D'souza ${ }^{1}$, Haidar F. AL-Qrimli², Omar Dhari Hussein ${ }^{3}$ \\ ${ }^{1}$ Department of Mechanical Engineering, Curtin University Sarawak Malaysia. \\ ${ }^{2}$ Studies Planning and Follow-up Department, MRC, Ministry of Oil, Baghdad, Iraq \\ ${ }^{3}$ Technical Directorate, Ministry of Oil, Baghdad, Iraq \\ Email: ${ }^{2}$ halqrimli@yahoo.com
}

\begin{abstract}
This article presents a hybrid design for a quadrotor aircraft. The hybrid is a combined system of a land robot and a quadrotor aircraft aimed at performing both driving and flying tasks. The hybrid quadrotor performs flying tasks in the usual way a normal quadrotor would do while the added caterpillar tracks would help maneuver over any type of terrain. The current prototype design was established after analyzing multiple designs and their response to various loads and impact forces. This study focuses on a practical approach to designing a hybrid quadrotor with experimental studies demonstrating the performance and feasibility on both flying and driving missions. The research outcomes were successful, and the new hybrid structure managed to survive a drop from a height greater than 2 meters. The hybrid quadrotor prototype was tested using finite element analysis and validated experimentally.
\end{abstract} track

Keywords - hybrid, quadrotor, drone, flying robot, caterpillar

\section{INTRODUCTION}

As of the last few years, studies on Unmanned Aerial Vehicles (UAVs) has dramatically increased. With advances in technology, the capabilities of UAVs have steadily improved making them more useful and creating multiple uses for them. Its uses range from hobby enthusiasts to oil and gas applications. Quite recently, UAVs have been used in a more commercial environment to deliver packages to customers, to record videos from unique angles for advertising agencies and in certain off-road sports or even events [1-2]. But the appearance of COVID-19 and other contagious disease, the future is going more rapidly towards autonomous technology. Most UAVs have a standard Takeoff and landing (CTOL) system which is acceptable in certain situations high altitude surveillance which would not be cramped for space during take-off or landing procedures nor would it require to maintain a certain geographical position based on global positioning [3-6].

While this is acceptable in most scenarios, certain situations such as low altitude surveillance in urban areas or maneuvering within a building requires increased maneuverability and hovering capabilities. The ability to take-off and land vertically (VTOL), to be able to hover and maintain its position became a priority. Vehicles with the VTOL system such as the helicopter have existed for quite some time [7-10]. From this single rotor design has led to a new subsection of UAVs with VTOL capabilities known as multi-rotors. While the helicopter is capable of taking off and landing vertically, it is not the most versatile aircraft and has difficulty in changing directions suddenly or achieving a high speed. Also, the helicopter was not designed to be an omnidirectional aircraft, so for example, moving backwards becomes a difficult maneuver to control and can be dangerous. Within tight spaces in an urban environment such as between buildings or under structures such as bridges or in tunnels, being omnidirectional would be a great asset to have. Multi-rotors aim to address these issues while improving on the overall design on a VTOL system [11-12].

Among the various types of multi-rotors, the quadrotor, as its name suggests uses a four-rotor system to fly. A four-rotor system has many advantages over other available systems. Capabilities such as hovering while maintaining direction becomes far less complicated when compared to a Single rotor design. Also, maneuvering during flight is done by controlling the RPM of each motor as opposed to varying the angles of the blades in a single rotor system which is relatively more difficult. Since maneuvering is done by controlling the rpm of each motor rather than controlling the angle of the blades, it makes the aircraft more capable in being omnidirectional. Also, because of the increase in rotors, the quadrotor is capable of lifting a much heavier payload as compared to a single rotor [13-15].

In recent years, quadrotor systems being developed have focused on system control [16], maneuverability [18] and autonomous navigation through collaboration with multiple UAVs [19]. These research topics focus on the quadrotors flying capabilities. A hybrid version of a quadcopter, one that is capable of flying and driving is rarely considered for research [20-24]. While UAVs and UGVs are very capable in their own respected ways, they are limited as to what they can do. A UAV is capable of traversing long distances in a very short amount of time but when it comes to performing any type of work in a specific place, it fails in comparison with other types of unmanned vehicles. When in close quarters with terrain, wildlife, people or anything else, it becomes difficult to control and could prove to be dangerous to others or even itself. Carbon fiber and other composite materials was used to reduce the load ratio in different structures and main body of the UAVs [25-27]. An Unmanned Ground Vehicle (UGV) would be more capable than quadcopters at certain 
tasks that require the vehicle to be in proximity or requires precision handling of an organism or substance.

Therefore, in this article, an innovative and unique hybrid quadrotor design, capable of flying and driving is presented. The design concept for this hybrid is as shown in Figure 1. While the four motors normally present in a quadrotor handle flying capabilities, two additional motors are added to support driving capabilities. For driving capabilities, two motorized gears are used on the left and right side of the quadrotor, each of which drive a continuous track, a similar system to that of a tank. Just as in a tank, changing directions is done by creating a difference in RPM between the motors.

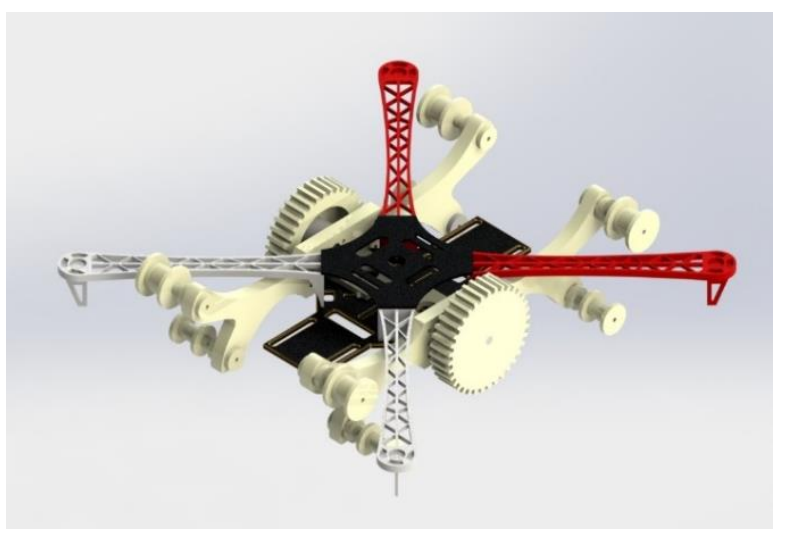

Fig. 1. Design Concept for a hybrid quadrotor

The uniqueness of this design is that theoretically, it can be implemented on any existing quadrotor platform. Simulation and experimental studies on both flying and driving ability are conducted to demonstrate the feasibility of this concept design.

\section{OPERATION OF A HYBRID QUADROTOR SYSTEM}

\section{A. Flying operations}

The hybrid quadrotor follows a standard symmetrical ' $\mathrm{X}$ ' layout for the four motors, with adjacent motors rotating in opposite directions, one rotating clockwise with the other rotating anti-clockwise to counter each other's moment on the center of the system. This ensures that the hybrid quadrotor does not spin out of control. In order to maneuver in any direction, the rpm of individual motors is manipulated to create the desired movement, as shown in Figure 2. This is what makes a quadrotor exceptional at omnidirectional movement. Table 1 describes the operation system for a quadrotor and how it maneuvers in any direction. This system applies to the Hybrid Quadrotor mentioned in this article as well.

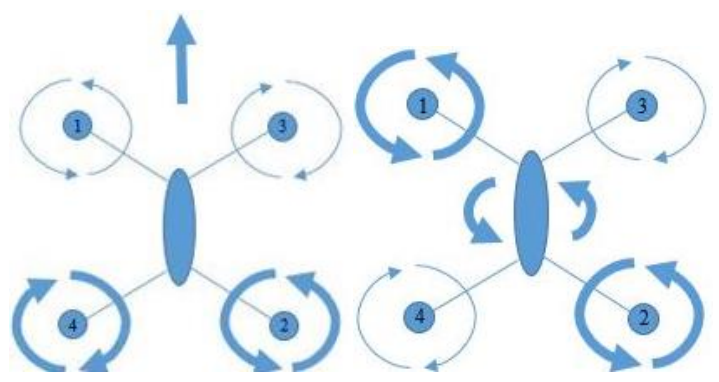

Fig. 2. Forward directional movement (Left) and anti-clockwise rotational movement (Right)
TABLE I. LATERAL AND ROTATIONAL MOVEMENT THROUGH ROTOR RPM MANIPULATION FOR A QUADROTOR

\begin{tabular}{|c|c|c|c|c|}
\hline \multirow{2}{*}{ Move up } & \multicolumn{4}{|c|}{ Rotor RPM Manipulation } \\
\cline { 2 - 5 } & Rotor 1 & Rotor 2 & Rotor 3 & Rotor 4 \\
\hline Move Down & Decrease & Increase & Increase & Increase \\
\hline Hold Position & Maintain & Maintain & Maintain & Maintain \\
\hline Move Forward & Maintain & Increase & Maintain & Increase \\
\hline Move Backward & Increase & Maintain & Increase & Maintain \\
\hline Roll Left & Maintain & Increase & Increase & Maintain \\
\hline Roll Right & Increase & Maintain & Maintain & Increase \\
\hline Yaw Left & Increase & Increase & Maintain & Maintain \\
\hline Yaw Right & Maintain & Maintain & Increase & Increase \\
\hline
\end{tabular}

\section{B. Driving operations}

Utilizing the same concept for movement in a tank, the two continuous tracks on each side of the quadrotor work together to perform driving maneuvers as shown in Figure 3. By either creating a difference in RPM between the motors or by rotating them in opposite directions, maneuvers such as turning left and right or rotating while maintaining its current position is possible. Table 2 describes the operation system for a vehicle with continuous tracks on how it maneuvers in any direction. This system applies to the Hybrid Quadrotor mentioned in this article as well.

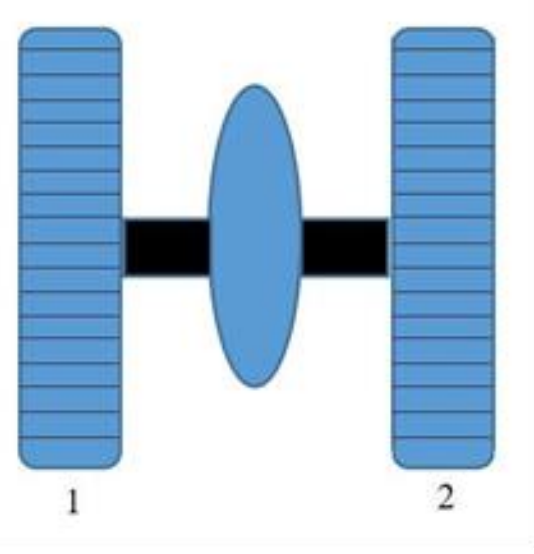

Fig. 3. Layout for a vehicle with continuous tracks

TABLE II. MOTOR RPM MANIPULATION MOVEMENT FOR VEHICLE WITH CONTINUOUS TRACKS

\begin{tabular}{|c|c|c|}
\hline \multirow{2}{*}{} & \multicolumn{2}{|c|}{ Rotor RPM Manipulation } \\
\cline { 2 - 3 } Move Forward & Rotor 1 & Rotor 2 \\
\hline Move Backward & Reverse & Reverse \\
\hline Turn Left & Decrease & Increase \\
\hline Turn Right & Increase & Decrease \\
\hline Rotate Left & Reverse & Forward \\
\hline Rotate Right & Forward & Reverse \\
\hline
\end{tabular}




\section{Control System}

The avionics system is the brain of the quadrotor and helps navigate and control the system. It is in charge of controlling the current to the motors, monitors the IMU and reads current information about the UAV such as altitude, orientation, velocity and direction of movement. With the exception of the two motors that are directly connected to the radio receiver, Figure 4 shows the standard hardware layout for a quadrotor build.

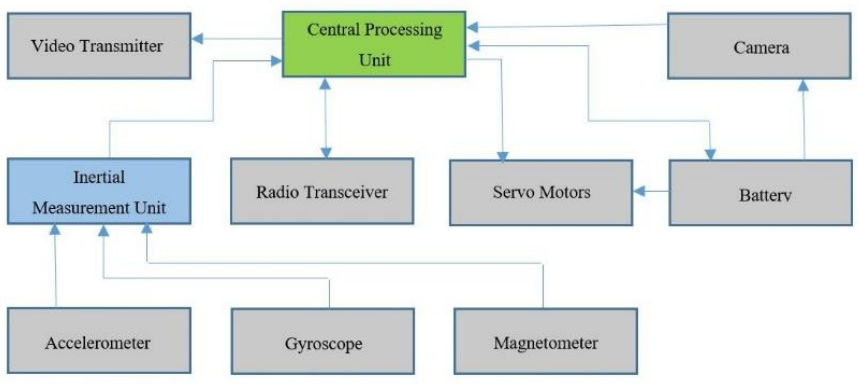

Fig. 4. Standard Avionics System for an unmanned aircraft

The Hybrid Quadrotor modelled in this article uses an 8channel radio receiver for the system. The first four channels are used for flight controls: Pitch, Throttle, Yaw and Roll. As seen in Figure 5, the two motors for the continuous tracks are directly connected to the radio receiver on channels 7 and 8 . The radio controller used for this hybrid quadrotor was a FLYSKY FS-TH9X radio.

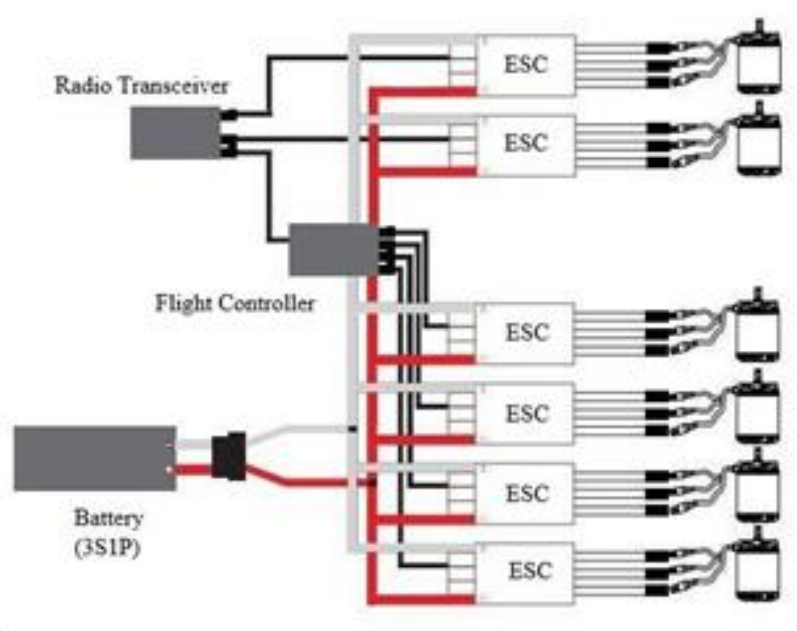

Fig. 5. Standard Layout for electronics for the Hybrid Quadrotor

\section{DESIGN AND SIMULATION OF THE HYBRID ADD-ON FOR THE QUADROTOR}

The prototype shown in Figure 6 was the chosen design for building the hybrid quadrotor. The material chosen for the prototype was Acrylonitrile Butadiene Styrene (ABS) plastic. The ABS plastic used to 3D print the prototype has very slight elastic properties with a yield strength of $4.25 \mathrm{e} 7 \mathrm{~N} / \mathrm{m} 2$ [2830].

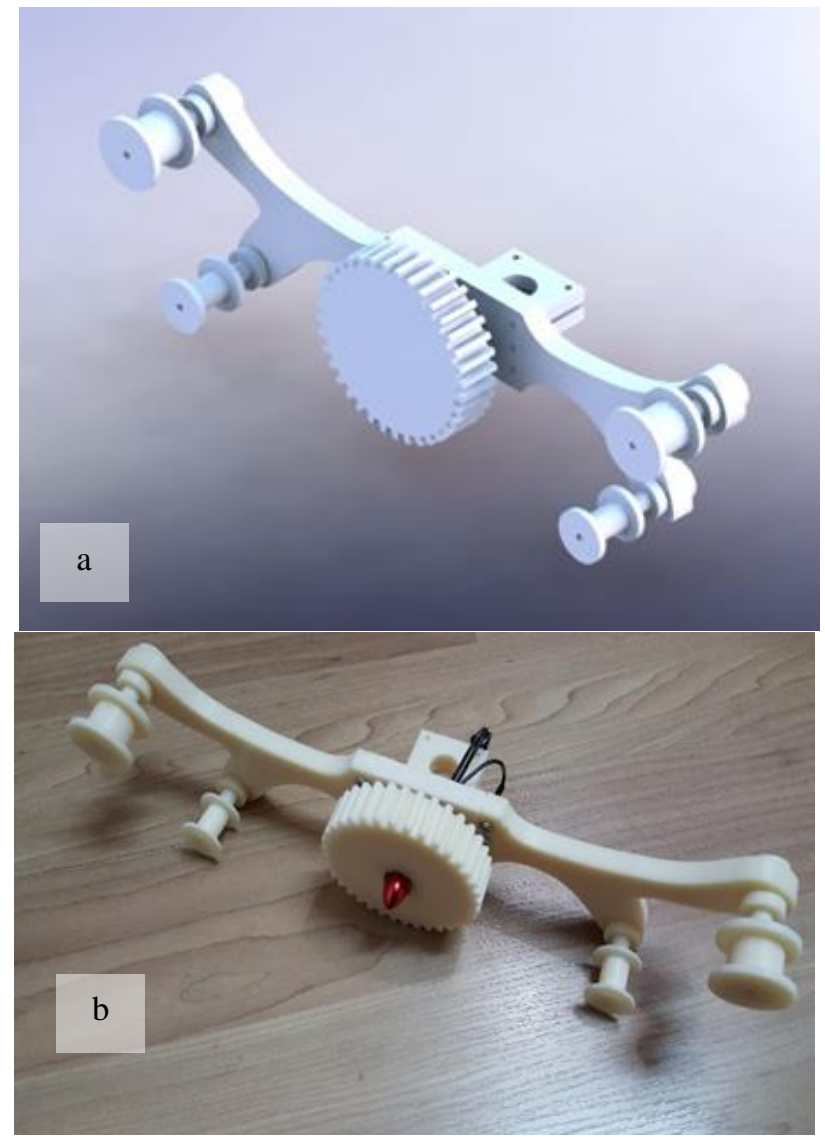

Fig. 6. 3D render of the chosen design for the hybrid quadrotor (a); Assembled model of the prototype design (b)

\section{A. Static Load Test}

Based on the estimated weight of $3 \mathrm{Kg}$ for the final quadrotor, a static load simulation in which the center part of the model attached to the quadrotor was kept fixed and a load of $750 \mathrm{~N}$ was applied to the base of the model. This was to simulate a drop from $5 \mathrm{~m}$. The impact velocity was found to be $9.91 \mathrm{~m} / \mathrm{s}$. While the frame was able to absorb most of the energy from impact, the stress concentrations on the center of the arm were too high. This would lead to plastic deformation and possible fractures or breaks (Figure 7).

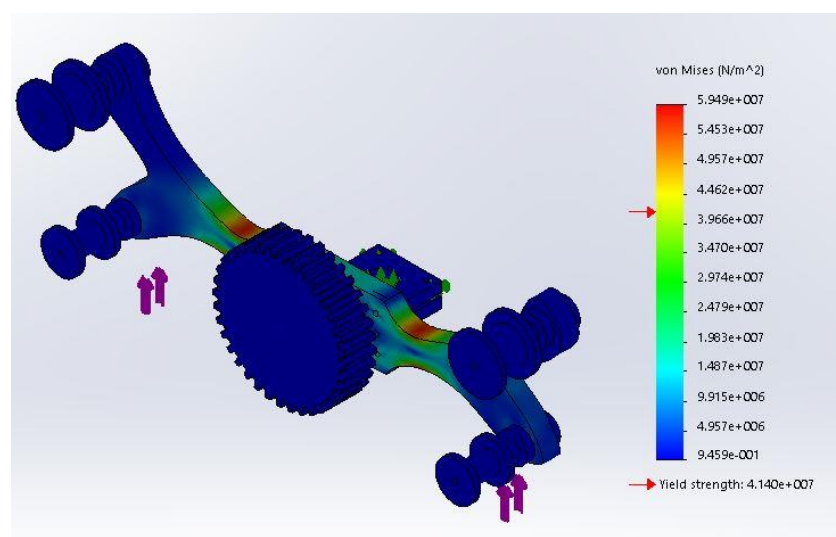

Fig. 7. 3D Static load test on the prototype 


\section{B. Drop Test}

To simulate a drop test, a stopping distance of $10 \mathrm{~mm}$ was assumed. A vertical orientation for the prototype as assumed and the floor was horizontal. The effects of von Mises stress, axial stress and stress intensity acting on the specimen was studied over the first 600 microseconds from impact. Figure 8 shows the effect of these stresses over the simulation period.

Based on the simulations, the ABS plastic cannot survive a drop from a height greater than 2 meters. Correspondingly, these results are based on ideal conditions. However, real tests confirmed higher values for stress. The quadrotor succeeded and survived a 2 meters height during experimental drop tests. But it should be mentioned that constant exposure to these stresses may cause fatigue and lower the life of the prototype.
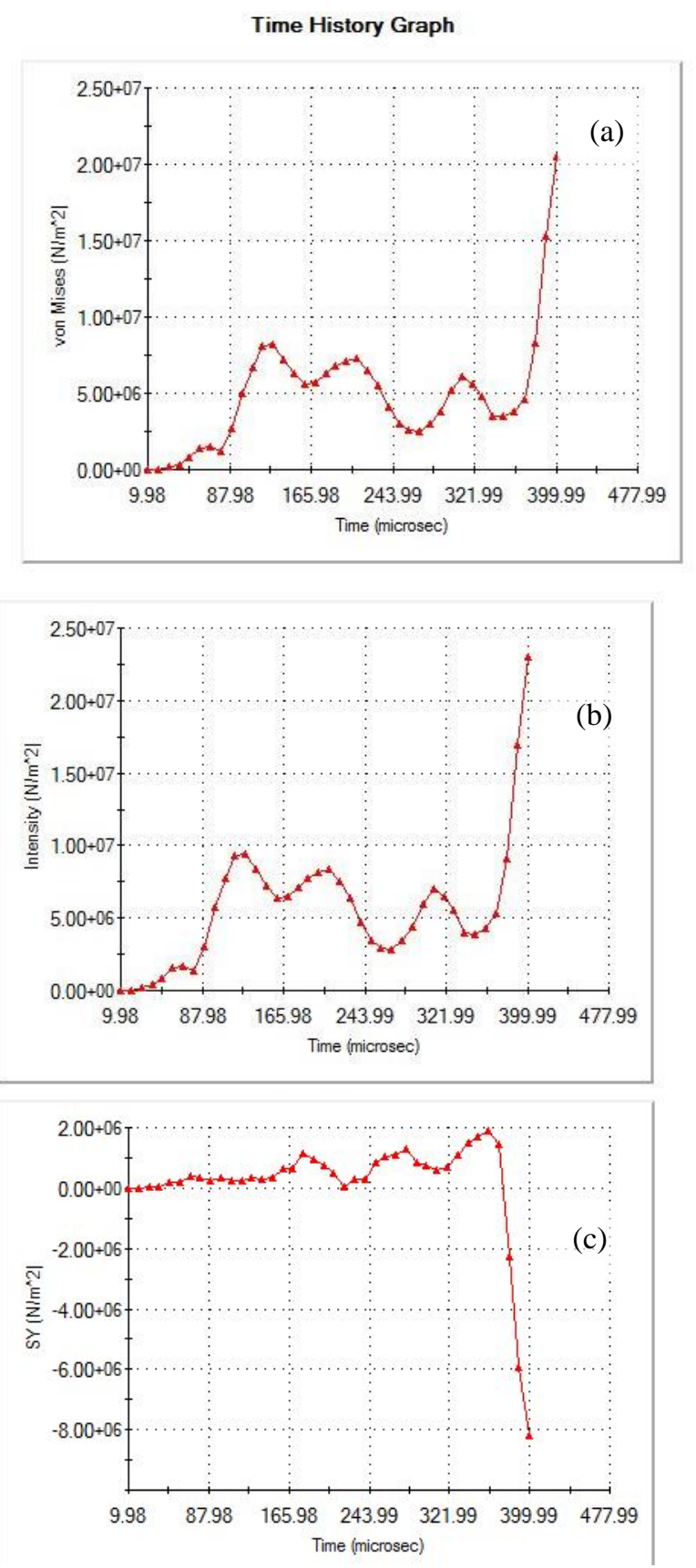

Fig. 8. (a) Von Mises Stress, (b) Stress intensity and (c) Axial Stress

\section{Flight TEST}

After constructing the prototype, a flight test revealed no negative effects on the quadrotor's flying capabilities. The quadrotor had no trouble getting off the ground or during any of its flight maneuvers. In driving mode as well, the caterpillar tracks had no issues with interference with the rest of the quadrotor.

The quadrotor was flown to a height of 120 meters before it started to disappear out of sight. Any further would require video feedback from the quadrotor as to not damage or lose it. It had a total flight time of roughly 14 minutes. This was at varying levels of throttle and would probably be extended during normal use.

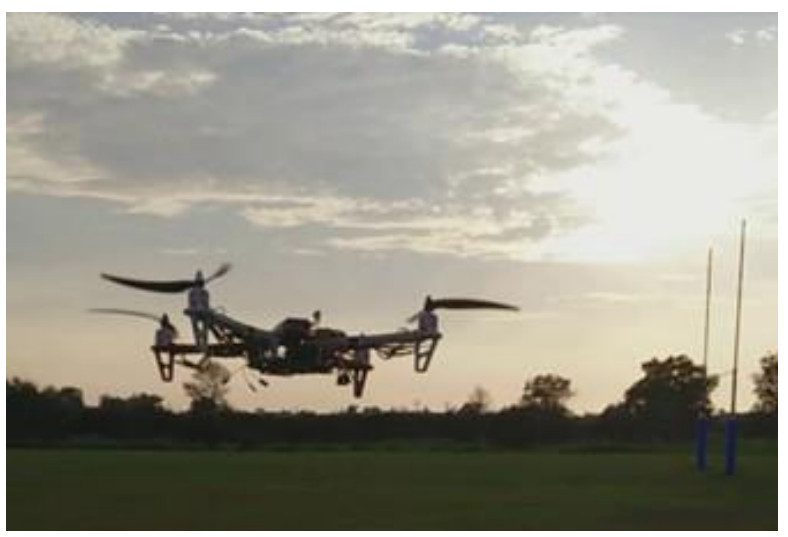

Fig. 9. Flight Test

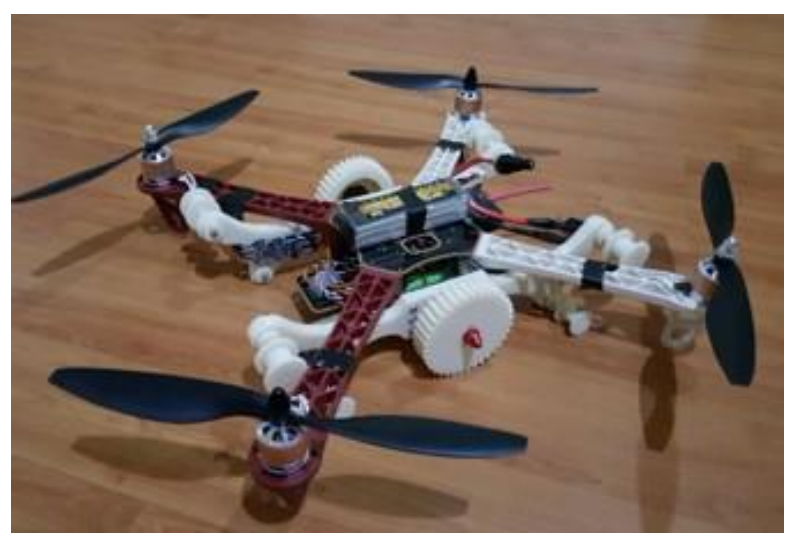

Fig. 10. Flight Test

\section{CONCLUSION}

The major achievement for this project was the design and construction of a new platform for hybrid quadrotors for future improvement and development. Recalling back to the this study's objectives, which were to investigate methods through which a quadrotor could be converted in to a hybrid, to be capable of travelling on rough terrain and to be able to maintain its structural integrity in case of sudden small impact forces such as from falling from a relatively low height by simulating its behavior accurately using Finite Element Analysis. The purpose of this study was to approach hybrid designs of quadrotors in a different way from what is currently being done. This project managed to successfully combine what is arguably the most versatile forms from both land and air vehicles and create a hybrid that could take 
advantage of the maneuverability of both these types of vehicles.

\section{REFERENCES}

[1] Alexis K, Nikolakopoulos G and Tzes A. Switching model predictive attitude control for a quadrotor helicopter subject to atmospheric disturbances. Control Eng Pract. 19, pp. 1195-1207, 2011.

[2] J. P. Campbell, "Vertical Takeoff and Landing Aircraft, "NASA Technical Reports Server (NTRS), 1962.

[3] Matko Orsag, Marina Poropat, Stjepan Bogdan, Hybrid Fly-by-Wire Quadrotor Controller, Automatika. 51 (1), 2010. DOI: 10.1109/ISIE.2010.5637585.

[4] Moussa Labbadi, Mohamed Cherkaoui, Robust adaptive nonsingular fast terminal sliding-mode tracking control for an uncertain quadrotor UAV subjected to disturbances, ISA Transactions, ISA Transactions. 99, Pages 290-304, 2020. DOI: 10.1016/j.isatra.2019.10.012.

[5] Ning Wang, Qi Deng, Guangming Xie, Xinxiang Pan, " Hybrid finitetime trajectory tracking control of a quadrotor, "ISA Transactions, Volume 90, pp. 278-286, 2019.

[6] Luis Alberto Paramo Carranza, E.C. Garcia, Jesus Alberto Meda Campaña, Quadrotor stabilization by Fuzzy Kalman Filter, Journal of Intelligent and Fuzzy Systems 38(4):4485-4494, 2020. DOI: 10.3233/JIFS-191251.

[7] Gessow and G. C. Mayers, Jr., Aerodynamics of the helicopter. New York, USA, Frederick Ungar Publishing, 1967.

[8] M. Achtelik, T. Zhang, K. Kuhnlenz and M. Buss, "Visual tracking and control of a quadcopter using a stereo camera system and inertial sensors, "Mechatronics and Automation, pp. 2863-2869, 2009.

[9] Ozdemir U, Aktas YO, Vuruskan A, Design of a commercial hybrid VTOL UAV system. J Intell Robot Syst. 74. pp. 371-393, 2014.

[10] H. Kim and D. J. Lee, "Real-Time Flight Testing for Developing an Autonomous Indoor Navigation System for a Multi-Rotor Flying Vehicle," The Korean Society of Mechanical Engineers, pp. 343-352, 2016.

[11] Hang Zhang, Bifeng Song, Haifeng Wang, Jianlin Xuan, A method for evaluating the wind disturbance rejection capability of a hybrid UAV in the quadrotor mode, International Journal of Micro Air Vehicles 11:175682931986964, 2019. DOI: 10.1177/1756829319869647.

[12] Zhenyu Tang, Hao Li, Junfeng Wang, Qinghai Liu, Hua Zhao, Xiaojun Guo, " Design and Test of the Propulsion System of a Mars Quadrotor UAV," Signal and Information Processing, Networking and Computerspp pp. 270-277, 2020.

[13] Mizouri, S. Najar, L. Bouabdallah, Mohamed Aoun, Dynamic Modeling of a Quadrotor UAV Prototype, Springer, pp 281-299, 2020. DOI: 10.1007/978-981-15-1819-5_14.

[14] Shi D, Wu Z and Chou W. Super-twisting extended state observer and sliding mode controller for quadrotor UAV attitude system in presence of wind gust and actuator faults. Electronics. 7, pp- 1-21, 2018.

[15] R. Hoogervorst, S. Stramigioli, H. W. Wopereis and M. Fumagalli, "Vision-IMU based collaborative control of a blind UAV," Workshop on Research, Education and Development of Unmanned Aerial Systems, pp. 53-61, 2015.

[16] Tiehua Wang, Kazuki Umemoto, Takahiro Endo, Fumitoshi Matsuno, Dynamic hybrid position/force control for the quadrotor with a multi- degree-of-freedom manipulator, Artificial Life and Robotics 24(3), 2019. DOI: 10.1007/s10015-019-00534-0.

[17] Zhen Xu, Xin-Zhi Liu, Qing-Wei Chen, Hunting problems of multiquadrotor systems via bearing-based hybrid protocols with hierarchical network, Chinese Physical Society and IOP Publishing Ltd. 2020.

[18] S. H. Jeong and S. Jung, "A quad-rotor system for driving and flying missions by tilting," ScienceDirect, pp. 1178-1188, 2014.

[19] Ju-Qian Zhang, Yu-Ru Shi, Yong Wu, Bang-Chun Wen, Nonlinear Hybrid Controller Design for Perturbed Quadrotor Robot by Uncertainties, International Journal of Precision Engineering and Manufacturing. 19 (11), pp. 1637-1650, 2018. DOI: 10.1007/s12541018-0191-z.

[20] Saeed AS, Younes AB, Cai C, A survey of hybrid unmanned aerial vehicles, Prog Aerosp Sci. 98, pp. 91-105, 2018.

[21] Zhang, B, Tang, Q;Zhang, R.; Chen, L.; Xu, M.; Yi, T., Droplets movement and deposition of an eight-rotor agricultural UAV in downwash flow field”. Int. J. Agric. Biol. Eng. 10, pp. 47-56, 2017.

[22] Haidar F. Al-Qrimli, KS Khalid, AM Abdelrhman, RK Mohammed, HM Hadi, A review on a straight bevel gear made from composite, Journal of Materials Science Research 5 (3), 73-82, 2016.

[23] ABS Data Sheets," [Online]. Available: http://teststandard.com/data_sheets/ABS_Data_sheet.pdf. [Accessed 2 2016].

[24] Kamal A and Ramirez-Serrano A, Development of a preliminary design methodology for transitional UAV, AIAA Aerospace Sciences Meeting, Kissimmee, Florida, pp. 1-15, 2018.

[25] HF AL-Qrimli, FA Mahdi, FB Ismail, Carbon/epoxy woven composite experimental and numerical simulation to predict tensile performance, Advances in Materials Science 4 (2), 33-41, 2015. DOI: 10.5963/AMSA0402001.

[26] Mahony R, Kumar V and Corke P., Multirotor aerial vehicles: modeling, estimation, and control of quadrotor, IEEE Robot Autom Mag. 19, pp. 20-32, 2012.

[27] Adigbli, C. Grand, J. B. Mouret and S. Doncieux, "Nonlinear Attitude and Position Control of a Micro Quadrotor using Sliding Mode and Backstepping Techniques". World Academy of Science, Engineering and Technology. 26, 2007.

[28] J. Yang, C.-X. Wang, Z.-S. Yu, Y. Li, K.-K. Yang, and Y.-Z. Wang, Impact behavior and fracture morphology of acrylonitrile-butadienestyrene resins toughened by linear random styrene-isoprene-butadiene rubber, J. Appl. Polym. Sci. 121, pp. 2458-2466, 2011. doi:10.1002/app.33773.

[29] Haidar F. Al-Qrimli, HSS Aljibori, Energy Absorption of Thin-Walled Composite Tubes Subjected under Quasi-static Compression, IEEE second international conference on Computer and Network Technology, (2010) 545-549. DOI: 10.1109/ICCNT.2010.101.

[30] X. Y. Xu and X. F. Xu, Mechanical properties and deformation behaviors of acrylonitrile-butadiene-styrene under Izod impact test and uniaxial tension at various strain rates, Polym. Eng. Sci. 51, pp. 902907, 2011. DOI:10.1002/pen.21908. 